\title{
Recent Developments in Distribution Theory: A Brief Survey and Some New Generalized Classes of distributions
}

\author{
Zubair Ahmad \\ Department of Statistics, Yazd University, \\ Yazd, Iran \\ z.ferry21@gmail.com \\ G. G. Hamedani \\ Department of Mathematics, Statistics and Computer Science, Marquette University, \\ WI 53201-1881, Milwaukee, USA \\ gholamhoss.hamedani@marquette.edu \\ Nadeem Shafique Butt \\ Department of Family and Community Medicine, \\ King Abdulaziz University \\ nshafique@kau.edu.sa
}

\begin{abstract}
The generalization of the classical distributions is an old practice and has been considered as precious as many other practical problems in statistics. These generalizations started with the introduction of the additional location, scale or shape parameters. In the last couple of years, this branch of statistics has received a great deal of attention and quite a few new generalized classes of distributions have been introduced. We present a brief survey of this branch and introduce several new families as well.
\end{abstract}

Keyword: Generalized classes of distributions; Exponentiated family; Marshall Olkin family; Transmuted family; Kumaraswamy family; Alpha power transformation; ZubairG family; Construction of new families.

\section{Introduction}

The recent development in distribution theory stresses on problem solving faced by the researchers and proposes a variety of models so that lifetime data sets can be better assessed and investigated in different applied areas. In other words, there is a need to introduce useful models for the better exploration of the real phenomenon of nature. Nowadays, the trends and practices in proposing new probability models totally differ in comparison to the models suggested before 1997. One main objective for proposing, extending or generalizing (models or their classes) is to explain how the lifetime phenomenon arises in fields like physics, computer science, insurance, public health, medical, engineering, biology, industry, communications, life-testing and many others. The well-known and fundamental distributions such as exponential, Rayleigh, Weibull and gamma are very limited in their characteristics and are unable to show wide flexibility. For example, the exponential distribution is capable of modeling with constant hazard function, whereas, the Rayleigh distribution has increasing hazard function only. However, the Weibull is much flexible and capable of modeling with increasing, decreasing or constant hazard function. 
Unfortunately, the Weibull model is not capable of modeling with non-monotonic (such as unimodal, modified unimodal or bathtub shaped) failure rate function. The gamma distribution does not have a closed form of cumulative distribution function (cdf) which causes difficulties in describing its mathematical properties. For complex phenomenon in human mortality studies, reliability studies, lifetime testing, engineering modeling, electronic sciences and biological surveys, the failure rate behavior can be bathtub, upsidedown bathtub and other shaped but not usually monotone increasing or decreasing. Thus, in order to cope with both monotonic and non-monotonic failure rate shapes, researchers have proposed several generalized classes of distributions which are very flexible to study needful properties of the model and its fitness. In the last two decades, several generalization approaches were adopted and practiced, which have received increased attention.

The objectives of the present study are three-fold: Firstly, we present an up-to-date account of the extended classes of distributions for the readers of modern distribution theory. Secondly, this survey will motivate the researchers to fill up the gap and to furnish their work in remaining applied areas. Thirdly, we propose some new classes of distributions which might be helpful as a tutorial to the beginners of the generalized modeling art.

The rest of the article is organized as follows. In Section 2, some extended classes of distributions are reviewed. In section 3, we present some new families. Section 4 presents certain characterizations of the distributions listed in Section 3. Finally, concluding remarks are provided in Section 5.

\section{Review of the existing family of distributions}

In this section, we present up-to-date review of the extended families of distributions.

\subsection{The exponentiated family of distributions}

Mudholkar and Srivastava (1993) proposed another method of introducing an extra parameter to a two-parameter Weibull distribution. The cumulative distribution function of the Mudholkar and Srivastava (1993)'s proposed exponentiated family has the following form

$$
G(x ; \alpha, \xi)=F(x ; \xi)^{a}, \quad \alpha, \xi>0, \quad x \in \mathbb{R},
$$

where $\alpha>0$ is an extra shape parameter. Due to the presence of an extra shape parameter, the proposed exponentiated distributions are more flexible than the traditional models. Using (1), a number of modifications of the existing distributions have been proposed in the literature. A brief list of these modifications is presented in Table 1: 
Table 1: Contributed work on exponentiated distributions

\begin{tabular}{|c|c|c|c|}
\hline S. No. & Year & Distribution & Author(s) \\
\hline 1 & 2001 & Exponentiated Exponential & Gupta and Kundu (2001) \\
\hline 2 & 2005 & Exponentiated beta & Nadarajah (2005) \\
\hline 3 & 2005 & Exponentiated Pareto & Nadarajah (2005) \\
\hline 4 & 2006 & exponentiated lognormal & Shirke and Kakde (2006) \\
\hline 5 & 2006 & exponentiated Fréchet & Nadarajah and Kotz (2006) \\
\hline 6 & 2006 & eexponentiated Gumbel & Nadarajah (2006) \\
\hline 7 & 2007 & exponentiated Gamma & Nadarajah and Gupta (2007) \\
\hline 8 & 2011 & exponentiated generalized gamma & Cordeiro et al. (2011) \\
\hline 9 & 2013 & exponentiated Lomax Poisson & Ramos et al. (2013) \\
\hline 10 & 2013 & exponentiated modified Weibull extension & Sarhan and Apaloo (2013) \\
\hline 11 & 2013 & exponentiated generalized class & Cordeiro et al. (2013) \\
\hline 12 & 2016 & exponentiated Weibull-Pareto & Afify et al. (2016) \\
\hline 13 & 2013 & exponentiated Kumaraswamy & Lemonte et al. (2013) \\
\hline 14 & 2014 & Exponentiated Kumaraswamy-Dagum & Huang and Oluyede (2014) \\
\hline 15 & 2014 & Exponentiated Half-Logistic family & Cordeiro et al. (2014) \\
\hline 16 & 2015 & Exponentiated Power Lindley & Ashour and Eltehiwy (2015) \\
\hline 17 & 2015 & Exponentiated power Lindley & Ashour and Eltehiwy (2015) \\
\hline 18 & 2015 & exponentiated generalized modified Weibull & Aryal and Elbatal (2015) \\
\hline 19 & 2015 & Exponentiated Burr XII Poisson & da Silva et al. (2015) \\
\hline 20 & 2015 & Exponentiated Generalized Gumbel & Andrade et al. (2015) \\
\hline 21 & 2015 & exponentiated transmuted generalized Rayleigh & Nofal et al. (2015) \\
\hline 22 & 2015 & exponentiated flexible Weibull extension & El-Gohary et al. (2015) \\
\hline 23 & 2016 & Exponentiated Gumbel Type-2 & Okorie et al.(2016) \\
\hline 24 & 2016 & Exponentiated Gompertz Generated Family & Cordeiro et al. (2016) \\
\hline 25 & 2017 & Exponentiated Generalized Weibull Gompertz & El-Bassiouny et al. (2017) \\
\hline 26 & 2017 & Exponentiated power Lindley Poisson & Pararai et al. (2017) \\
\hline 27 & 2017 & $\begin{array}{l}\text { Exponentiated inverse flexible Weibull } \\
\text { extension }\end{array}$ & $\begin{array}{l}\text { Morshedy and El-Bassiouny } \\
\text { (2017) }\end{array}$ \\
\hline 28 & 2017 & Exponentiated Lomax Geometric & Hassan and Abd-Allah (2017) \\
\hline 29 & 2018 & Exponentiated Inverse Power Lindley & Jan et al. (2018) \\
\hline 30 & 2018 & Exponentiated Weibull-Lomax & Hassan and Abd-Allah (2018) \\
\hline
\end{tabular}

\subsection{The Marshall-Olkin family of distributions}

Marshall and Olkin (1997) pioneered a simple method of adding a single parameter to a family of distributions and several authors used their method to extend well-known distributions in the last few years. If $\bar{F}(x ; \xi)$ and $F(x ; \xi)$ denote the survival function (sf) and cumulative distribution function of a parent distribution depending on the vector parameter $\xi$, then the sf of Marshall and Olkin (MO) family is defined by

$\bar{G}(x ; \sigma, \xi)=\frac{\sigma \bar{F}(x ; \xi)}{1-\bar{\sigma} \bar{F}(x ; \xi)}, \quad \quad \xi, \sigma>0, x \in \mathbb{R}$, 
Ahmed, Z., Hamedani, G. G. and Butt, N.S.

where, $\bar{\sigma}=1-\sigma$. Clearly, for $\sigma=1$, we obtain the baseline distribution, i.e., $\bar{F}(x ; \xi)=\bar{G}(x ; \xi)$

. Using (2), the extended versions of the existing distributions have been proposed. Based on the MO family, a detail review of the existing distributions is provided in Table 2:

Table 2: Contributed work on Marshall-Olkin distributions

\begin{tabular}{|c|c|c|c|}
\hline $\begin{array}{l}\text { S. } \\
\text { No. }\end{array}$ & Year & Distribution & Author(s) \\
\hline 1 & 2003 & Marshall Olkin Pareto & Alice and Jose (2003) \\
\hline 2 & 2005 & Marshall Olkin extended Pareto & Ghitany (2005) \\
\hline 3 & 2005 & Marshall Olkin semi Weibull & Alice and Jose (2005) \\
\hline 4 & 2005 & Marshall Olkin Logistic & Alice and Jose (2005) \\
\hline 5 & 2005 & Marshall Olkin extended Weibull & Ghitany et al. (2005) \\
\hline 6 & 2007 & Marshall-Olkin gamma & Ristic et al. (2007) \\
\hline 7 & 2007 & Marshall-Olkin extended Lomax & Ghitany (2007) \\
\hline 8 & 2009 & Marshall-Olkin beta & Jose et al. (2009) \\
\hline 9 & 2011 & Marshall-Olkin extended exponential & Rao et al. (2011) \\
\hline 10 & 2010 & Marshall Olkin q-Weibull & Jose et al. (2010) \\
\hline 11 & 2011 & Marshall-Olkin extended uniform & Jose and Krishnu (2011) \\
\hline 12 & 2013 & Marshall-Olkin Extended Log-Logistic & Gui (2013) \\
\hline 13 & 2013 & Marshall-Olkin Extended Zipf & Casany and Casellas (2013) \\
\hline 14 & 2013 & Marshall-Olkin power log-normal & Gui (2013) \\
\hline 16 & 2013 & Marshall-Olkin extended Weibull & Cordeiro and Lemonte (2013) \\
\hline 17 & 2014 & Marshall-Olkin extended Weibull family & Santos-Neto et al. (2014) \\
\hline 18 & 2014 & Marshall Olkin extended Burr type XII & Al-Saiari et al. (2014) \\
\hline 19 & 2014 & Marshall-Olkin discrete uniform & Sandhya and Prasanth (2014) \\
\hline 20 & 2015 & Marshall-Olkin generalized exponential & Ristic and Kundu (2015) \\
\hline 21 & 2015 & Marshall-Olkin exponential Weibull & Pogány et al. (2015) \\
\hline 22 & 2016 & Marshall-Olkin Extended Burr Type III & Kumar (2016) \\
\hline 23 & 2016 & Marshall-Olkin Flexible Weibull Extension & Mustafa et al. (2016) \\
\hline 24 & 2016 & Marshall-Olkin gamma-Weibull & Saboor and Pogány (2016) \\
\hline 25 & 2016 & Marshall-Olkin Additive Weibull & Afify et al. (2016) \\
\hline 26 & 2017 & Marshall-Olkin Extended Generalized Gompertz & Benkhelifa (2016) \\
\hline 27 & 2017 & $\begin{array}{l}\text { Marshall-Olkin Log-Logistic Extended Weibull } \\
\text { Marshall-Olkin generalized Erlang-truncated }\end{array}$ & Lepetu et al. (2017) \\
\hline 28 & 2017 & exponential & Okorie et al. (2017) \\
\hline 29 & 2017 & Marshall-Olkin Burr X family & Jamal et al. (2017) \\
\hline 30 & 2018 & Marshall-Olkin Extended Inverse Power Lindley & Hibatullah (2018) \\
\hline 31 & 2018 & Marshall-Olkin Extended Inverse Weibull & Pakungwati et al. (2018) \\
\hline 32 & 2018 & Marshall-Olkin Half Logistic & Yeğen and Özel (2018) \\
\hline 33 & 2018 & Marshall-Olkin generalized-G family & Yousof et al. (2018) \\
\hline
\end{tabular}

\subsection{Transmuted family of distributions}


Shaw and Buckley (2009) pioneered another prominent method of adding a parameter into a family of distributions and several authors used their method to extend well-known distributions in the last couple of years. If $F(x ; \xi)$ denotes the cdf of a parent distribution depending on the vector parameter $\xi$, then the cdf of the transmuted family is given by

$G(x ; \lambda, \xi)=(1+\lambda) F(x ; \xi)-\lambda F(x ; \xi)^{2}, \quad \xi>0,|\lambda| \leq 1, x \in \mathbb{R}$.

From (3), for $\lambda=0$, we obtain the baseline distribution, i.e., $F(x ; \xi)=G(x ; \xi)$. Using (3), the extended versions of the existing distributions have been proposed, for detail we refer to Tahir and Cordeiro (2016).

\subsection{Cubic Transmuted family of distributions}

Granzotto et al. (2017) proposed a new method of generating distributions called Cubic Transmutation method. Let $\mathrm{X}_{1}, \mathrm{X}_{2}$ and $\mathrm{X}_{3}$ be independent and identically random variables with distribution $F(x ; \xi)$. Then, the ranking cubic transmutation map is given by

$G(x ; \lambda, \xi)=\lambda_{1} F(x ; \xi)+\left(\lambda_{2}-\lambda_{1}\right) F(x ; \xi)^{2}+\left(1-\lambda_{2}\right) F(x ; \xi)^{3}, \quad \xi>0, x \in \mathbb{R}$,

with $\lambda_{1} \in[0,1]$ and $\lambda_{2} \in[-1,1]$.

Recently, Aslam et al. (2018) proposed Cubic transmuted-G family by using the T- $X$ idea of Alzaatreh (2013).

\subsection{A General Transmuted family of distributions}

Recently, Rahman et al. (2018) proposed a general transmuted family of distributions, is defined by

$G\left(x ; \lambda_{i}, \xi\right)=F(x ; \xi)+(1-F(x ; \xi)) \sum_{i=1}^{k} \lambda_{i} F(x ; \xi)^{i} \quad \xi>0, x \in \mathbb{R}$,

with $\lambda_{i} \in[-1,1]$ for $i=1 ; 2 ; \cdots ; k$ and $-k \leq \sum_{i=1}^{k} \lambda_{i} \leq 1$. The general transmuted family reduces to the base distribution for $\lambda_{i}=0$ for $i=1 ; 2 ; \cdots ; k$.

\subsection{Kumaraswamy-G family of distributions}

Kumaraswamy (1980) (for short Ku) proposed a two-parameter distribution on $(0,1)$, called Kumaraswamy distribution, is defined by

$G(x ; \alpha, \beta, \xi)=1-\left(1-x^{\alpha}\right)^{\beta}, \quad \xi>0, x \in(0,1)$,

where $\alpha>0$ and $\beta>0$ are shape parameters. The density function corresponding to (6) is

$g(x ; \alpha, \beta, \xi)=\alpha \beta x^{\alpha-1}\left(1-x^{\alpha}\right)^{\beta-1}, \quad x \in(0,1)$.

The $\mathrm{Ku}$ density has the same basic shape properties as to the beta distribution: $\alpha>1$ and $\beta>1$ (unimodal); $\alpha<1$ and $\beta<1$ (bathtub); $\alpha>1$ and $\beta \leq 1$ (increasing); $\alpha \leq 1$ and $\beta>1$ (decreasing) and $\alpha=\beta=1$ (constant). Using (7), for an arbitrary baseline distribution function $F(x ; \xi)$, Cordeiro and Castro proposed the cdf of the Kumaraswamy-G (Ku-G) family 
Ahmed, Z., Hamedani, G. G. and Butt, N.S.

$$
G(x ; \alpha, \beta, \xi)=1-\left(1-F(x ; \xi)^{\alpha}\right)^{\beta}, \quad \alpha, \beta, \xi>0, x \in \mathbb{R},
$$

Using (8), a number of modifications of the existing distributions have been proposed in the literature. A brief list of these modifications is presented in Table 3:

Table 3: Contributed work on Ku-G distributions.

\begin{tabular}{|c|c|c|c|}
\hline $\begin{array}{l}\text { S. } \\
\text { No. }\end{array}$ & Year & Distribution & Author(s) \\
\hline 1 & 2010 & Kumaraswamy Weibull & Cordeiro et al. (2010) \\
\hline 2 & 2011 & Kumaraswamy Generalized Gamma & Pascoa et al. (2011) \\
\hline 3 & 2012 & Kumaraswamy-Log-Logistic & Santana et al. (2012) \\
\hline 4 & 2012 & Kumaraswamy Pareto & Pereira et al. (2012) \\
\hline 5 & 2012 & Kumaraswamy Gumbel & Cordeiro et al. (2012) \\
\hline 6 & 2012 & Kumaraswamy Birnbaum-Saunders & Saulo et al. (2012) \\
\hline 7 & 2013 & Kumaraswamy Generalized Lomax & Shams (2013) \\
\hline 8 & 2013 & Kumaraswamy-Generalized Exponentiated Pareto & Shams (2013) \\
\hline 9 & 2013 & Kumaraswamy generalized linear failure rate & Elbatal (2013) \\
\hline 10 & 2013 & Kumaraswamy Pareto & Elbatal (2013) \\
\hline 11 & 2013 & Kumaraswamy Burr XII & Paranaba et al. (2013) \\
\hline 12 & 2013 & Kumaraswamy Generalized Pareto & Nadaraja and Eljabri (2013) \\
\hline 13 & 2014 & Kumaraswamy Inverse Rayleigh & Roges et al. (2014) \\
\hline 14 & 2014 & Kumaraswamy-geometric distribution & Akinsete et al. (2014) \\
\hline 15 & 2014 & Kumaraswamy modified Weibull & Cordeiro et al. (2014) \\
\hline 16 & 2014 & Kumaraswamy Lindley & $\begin{array}{l}\text { Merovci and Sharma } \\
\text { (2014) }\end{array}$ \\
\hline 17 & 2014 & Kumaraswamy Inverse Weibull & Shahbaz et al. (2014) \\
\hline 18 & 2014 & Kumaraswamy generalized Rayleigh & Gomes et al. (2014) \\
\hline 19 & 2014 & Kumaraswamy exponentiated Lomax & Elbatal and Kareem (2014) \\
\hline 20 & 2015 & Kumaraswamy Modified Inverse Weibull & Pararai et al. (2015) \\
\hline 21 & 2015 & Kumaraswamy Lindley-Poisson & Alizadeh et al. (2015) \\
\hline 22 & 2015 & Kumaraswamy odd log-logistic & Alizadeh et al. (2015) \\
\hline 23 & 2015 & Kumaraswamy Modified Inverse Weibull & Aryal and Elbatal (2015) \\
\hline 24 & 2016 & Kumaraswamy Gompertz Makeham & Chukwu and Ogunde (2016) \\
\hline 25 & 2016 & Kumaraswamy Laplace & Nassar (2016) \\
\hline 26 & 2016 & Kumaraswamy Exponentiated Inverse Rayleigh & Haq (2016) \\
\hline 27 & 2016 & Kumaraswamy transmuted-G & Afify et al. (2016) \\
\hline 28 & 2016 & Kumaraswamy Flexible Weibull Extension & El-Damcese et al. (2016) \\
\hline 29 & 2016 & Kumaraswamy exponential Weibull & Cordeiro et al. (2016) \\
\hline 30 & 2016 & Kumaraswamy generalized power Weibull & Selim and Badr (2016) \\
\hline 31 & 2016 & Kumaraswamy Weibull-G family & Hassan and Elgarhy (2016) \\
\hline 32 & 2016 & Kumaraswamy-Burr Type III & Behairy et al. (2016) \\
\hline 33 & 2017 & Kumaraswamy transmuted Pareto & Chhetri et al. (2017) \\
\hline 34 & 2017 & Inverted Kumaraswamy & AL-Fattah et al. (2017) \\
\hline 35 & 2017 & Kumaraswamy-Burr III & Kumar et al. (2017) \\
\hline 36 & 2017 & Kumaraswamy Inverse Exponential & Oguntunde et al. (2017) \\
\hline
\end{tabular}




\begin{tabular}{rrll}
\hline \multicolumn{1}{l}{ S. } & \multicolumn{1}{l}{ Year } & Distribution & Author(s) \\
\hline 37 & 2017 & Kumaraswamy transmuted exponentiated modified & \\
38 & 2017 & Kumaraswamy Half-Logistic & Al-Babtain et al. (2017) \\
39 & 2018 & Kumaraswamy Exponentiated U-Quadratic & Usman et al. (2017) \\
40 & 2018 & Kumaraswamy exponentiated Chen & Muhammad et al. (2018) \\
41 & 2018 & Kumaraswamy odd Burr-G family & Khan et al. (2018) \\
42 & 2018 & Kumaraswamy Marshall-Olkin Log-Logistic & Nasir et al. (2018) \\
\hline
\end{tabular}

\subsection{T- $X$ Family approach}

Eugene et al. (2002) introduced the beta generated method that uses the beta distribution with parameters $a$ and $b$ as the generator to develop the beta generated distributions. The distribution of a beta-generated random variable $X$ is defined as $G(x ; a, b, \xi)=\int_{0}^{F(x ; \xi)} r(t) d t, \quad a, b, \xi>0$

where $r(t)$ is the pdf of a beta random variable and $F(x ; \xi)$ is the cdf of any random variable $X$. Alzaatreh et al. (2013) proposed another method of generating families of continuous distributions called T- $X$ family by replacing the beta pdf with a pdf, $b(t)$, of a continuous random variable and applying a function $W\{F(x ; \xi)\}$ that satisfies some certain conditions.

Using the $\mathrm{T}-X$ idea, several new classes of distributions have been introduced in the literature. Table 4 provides some $W[F(x ; \xi)]$ functions for some members of the T- $X$ family.

\section{Table 4. Some members of the T- $X$ family}

\begin{tabular}{|c|c|c|}
\hline$W\{F(x ; \xi)\}$ & $\begin{array}{l}\text { Range of } \\
\quad T\end{array}$ & Members of $\mathrm{T}-X$ family \\
\hline$F(x ; \xi)$ & {$[0,1]$} & $\begin{array}{c}\text { Beta-G (Eugene et al., 2002), Mc-G (Alexander et } \\
\text { al., 2012) }\end{array}$ \\
\hline$-\log [F(x ; \xi)]$ & $(0, \infty)$ & $\begin{array}{l}\text { Gamma-G Type-2 (Risti'c and Balakrishnan, } \\
\text { 2012) }\end{array}$ \\
\hline$-\log [1-F(x ; \xi)]$ & $(0, \infty)$ & $\begin{array}{c}\text { Gamma-G Type-1 (Zografos and Balakrishnan, } \\
\text { 2009) }\end{array}$ \\
\hline$F(x ; \xi)$ & \multirow[b]{2}{*}{$(0, \infty)$} & \multirow[b]{2}{*}{ Gamma-G Type-3 (Torabi and Montazeri, 2012) } \\
\hline$\overline{1-F(x ; \xi)}$ & & \\
\hline$-\log \left[1-F^{\alpha}(x ; \xi)\right]$ & $(0, \infty)$ & Exponentiated T-X (Alzaghal et al., 2013) \\
\hline $\log \left\{\frac{F(x ; \xi)}{1-F(x ; \xi)}\right\}$ & $(-\infty \infty)$ & Logistic-G (Torabi and Montazeri, 2014) \\
\hline $\log [-\log \{1-F(x ; \xi)\}]$ & $(-\infty \infty)$ & The Logistic-X Family (Tahir et al., 2015) \\
\hline$\frac{-\log \{1-F(x ; \xi)\}}{1-F(x ; \xi)}$ & $(0, \infty)$ & New Weibull-X Family (Ahmad et al., 2018) \\
\hline
\end{tabular}


Ahmed, Z., Hamedani, G. G. and Butt, N.S.

\subsection{Alpha Power Transformation}

Mahdavi and Kundu (2017) proposed a new method for introducing statistical distributions via the cdf given by

$G(x ; \alpha, \xi)=\frac{\alpha^{F(x, \xi)}-1}{\alpha-1}, \quad \alpha, \xi>0, \alpha \neq 1, x \in \mathbb{R}$.

Using (10), some new extensions of the parent distributions have been introduced. A list of distributions based on alpha power transformation is provided in Table 5.

Table 5: Contributed work on alpha power transformation.

\begin{tabular}{cccc}
\hline $\begin{array}{c}\text { S. } \\
\text { No. }\end{array}$ & Year & Distribution & Author(s) \\
\hline 1 & 2017 & Alpha power exponential Weibull & Rahman and El-Bassiouny \\
& & & $(2017)$ \\
2 & 2018 & Alpha power inverted exponential & Unal et al. (2018) \\
3 & 2018 & Alpha power transformed Lindley & Dey et al. (2018) \\
4 & 2018 & Alpha power inverse Weibull & Ramadan and Walaa \\
& & & $(2018)$ \\
5 & 2019 & Alpha power transformed inverse Lindley & Dey et al. (2019) \\
6 & 2019 & Alpha power transformed Frechet & Nasiru et al. (2019) \\
7 & 2019 & Alpha power transformed power Lindley & Hassan et al. (2019) \\
\hline
\end{tabular}

\subsection{The Zubair-G family}

Recently, Ahmad (2018) proposed another method for generating new distributions via the cdf given by

$G(x ; \alpha, \xi)=\frac{e^{\alpha F(x ; \xi)^{2}}-1}{e^{\alpha}-1}, \quad \alpha, \xi>0, x \in \mathbb{R}$.

Using (11), some new modified versions of the parent distributions have been proposed. A list of distributions based on the Zubair-G method is provided in Table 6 .

Table 6: Contributed work on the Zubair-G family.

\begin{tabular}{cccc}
\hline $\begin{array}{c}\text { S. } \\
\text { No. }\end{array}$ & Year & Distribution & Author(s) \\
\hline 1 & 2019 & $\alpha-$ Zubair-G family & Kyurkchiev et al. \\
& & (2019) \\
2 & 2019 & Zubair-G distribution with baseline Lomax & Pavlov et al. (2019) \\
3 & 2019 & $\begin{array}{c}\text { Zubair-G distribution with baseline Ghosh- } \\
\text { Bourguignon's extended Burr XII }\end{array}$ & Rahneva et al. \\
& & (2019) \\
\hline
\end{tabular}




\section{New Proposed Families}

As we discussed in Section 2, the distribution theory has received serious consideration in the literature. We carry further this branch of statistics and propose some new methods for generating new distributions. We can define a general form of cdf via the expression

$G(x ; \xi)=\frac{e^{R(x ; \xi)}-1}{e-1}, \quad \xi>0, \quad x \in \mathbb{R}$,

where, $R(x ; \xi)$ is a baseline cdf. We can also take $R(x ; \xi)$ as any function of cdf, which obey the properties of cdf, or we may combine two or more distribution functions to propose a new class of distributions. For the sake of simplicity we omit the dependency on the vector parameter and we simply write $R(x ; \xi)=R(x), G(x ; \xi)=G(x)$ and $F(x ; \xi)=F(x)$. From (12), we can also define a new function as

$G(x)=\frac{e^{\alpha R(x)}-1}{e^{\alpha}-1}, \quad \alpha>0, \quad x \in \mathbb{R}$.

Taking $R(x)=F(x)^{2}$ in (13), we arrive at the Zubair-G distribution.

\subsection{The extended Zubair-G family}

In this sub-section, we define a new family of distributions, called the extended Zubair-G (EZ-G) family via taking $R(x)=\alpha F(x)^{2}+\beta F(x)$ in (12). The cdf of the EZ-G family is given by

$$
G(x)=\frac{e^{\alpha F(x)^{2}+\beta F(x)}-1}{e^{\alpha+\beta}-1}, \quad \alpha, \beta>0, \quad x \in \mathbb{R},
$$

where $\alpha>0$ and $\beta>0$ are the additional parameters. The density corresponding to (14) is

$$
g(x)=\frac{f(x)(2 \alpha F(x)+\beta) e^{\alpha F(x)^{2}+\beta F(x)}}{e^{\alpha+\beta}-1}, \quad x \in \mathbb{R} .
$$

Using (15), we can generate the extended version of the existing distributions. We discuss some special sub-models of the EZ-G class by considering $F(x ; \xi)$ as the cdf of the baseline model. In Table 7, we define $R(x)$ for the sub-models of the EZ-G class of distributions.

\section{Table 7: Special sub-models of the EZ-G family}

\begin{tabular}{ccccc}
\hline $\begin{array}{c}\text { S. } \\
\text { No. }\end{array}$ & Baseline model & $R(x)$ & Proposed model & Status \\
\hline 1 & Weibull & $\alpha\left(1-e^{-\eta x^{\gamma}}\right)^{2}+\beta\left(1-e^{-\eta x^{\gamma}}\right)$ & EZ-Weibull & New \\
\hline 2 & Lomax & $\alpha\left(1-(1+b x)^{-a-1}\right)^{2}+\beta\left(1-(1+b x)^{-a-1}\right)$ & EZ-Weibull & New \\
\hline 3 & uniform & $\alpha(x / \eta)^{2}+\beta(x / \eta)$ & EZ-uniform & New \\
\hline 4 & Exponential & $\alpha\left(1-e^{-\eta x}\right)^{2}+\beta\left(1-e^{-\eta x}\right)$ & EZ- exponential & New \\
\hline 5 & Rayleigh & $\alpha\left(1-e^{-\eta x^{2}}\right)^{2}+\beta\left(1-e^{-\eta x^{2}}\right)$ & EZ- Rayleigh & New \\
\hline
\end{tabular}


Ahmed, Z., Hamedani, G. G. and Butt, N.S.

\begin{tabular}{ccccc}
\hline 6 & $\begin{array}{c}\text { Linear failure } \\
\text { rate }\end{array}$ & $\alpha\left(1-e^{-\eta x^{2}-\gamma x}\right)^{2}+\beta\left(1-e^{-\eta x^{2}-\gamma x}\right)$ & $\begin{array}{c}\text { EZ- Linear failure } \\
\text { rate }\end{array}$ & New \\
\hline 7 & Pareto & $\alpha\left(1-(\eta /)^{a}\right)^{2}+\beta\left(1-(\eta /)^{a}\right)$ & EZ-Pareto & New \\
\hline 8 & Burr & $\alpha\left(1-\left(1+x^{b}\right)^{-a}\right)^{2}+\beta\left(1-\left(1+x^{b}\right)^{-a}\right)$ & EZ-Burr & New \\
\hline 9 & Topp-Leone & $\alpha\left(x^{b}\left(2-x^{b}\right)\right)^{2}+\beta\left(x^{b}\left(2-x^{b}\right)\right)$ & EZ-Topp-Leone & New \\
\hline
\end{tabular}

\subsection{The Cosine- $X$ family of distributions}

Taking $R(x)=1-\cos \left(\frac{\pi}{2} F(x)\right)$ in (9), we define the cosine- $X$ family as

$G(x)=\frac{e^{1-\cos \left(\frac{\pi}{2} F(x)\right)}-1}{e-1}, \quad x \in \mathbb{R}$,

The pdf corresponding to (16), is given by

$$
g(x)=\frac{\pi}{(e-1) 2} f(x) \sin \left(\frac{\pi}{2} F(x)\right) e^{1-\cos \left(\frac{\pi}{2} F(x)\right)}, \quad x \in \mathbb{R} .
$$

\subsection{The Cosine exponentiated- $X$ family of distributions}

A random variable $X$ is said to follow the Cosine exponentiated- $X$ distribution if its cdf is given by

$G(x)=\frac{e^{1-\cos \left(\frac{\pi}{2} F(x)^{a}\right)}-1}{e-1}, \quad a>0, x \in \mathbb{R}$,

with pdf

$$
g(x)=\frac{a \pi}{(e-1) 2} f(x) F(x)^{a-1} \sin \left(\frac{\pi}{2} F(x)^{a}\right) e^{1-\cos \left(\frac{\pi}{2} F(x)^{a}\right)}, \quad x \in \mathbb{R} .
$$

\subsection{The extended Cosine- $X$ family of distributions}

A random variable $X$ is said to follow the extended Cosine- $X$ (for short ' $\mathrm{EC}-X$ ') if its cdf is given by

$G(x)=\frac{e^{\alpha\left(1-\cos \left(\frac{\pi}{2} F(x)\right)\right)^{2}}-1}{e^{\alpha}-1}, \quad \alpha>0, x \in \mathbb{R}$,

with density function

$$
g(x)=\frac{\pi}{e^{\alpha}-1} f(x) \sin \left(\frac{\pi}{2} F(x)\right)\left(1-\cos \left(\frac{\pi}{2} F(x)\right)\right) e^{\alpha\left(1-\cos \left(\frac{\pi}{2} F(x)\right)\right)^{2}}, \quad \alpha>0, \quad x \in \mathbb{R} .
$$

\subsection{The extended Cosine exponentiated- $X$ family of distributions}

A random variable $X$ is said to follow the extended cosine exponentiated- $X$ (for short 'ECE$X^{\prime}$ ) distribution, if its cdf is given by 
$G(x)=\frac{e^{\alpha\left(1-\cos \left(\frac{\pi}{2} F(x)^{a}\right)\right)^{2}}-1}{e^{\alpha}-1}, \quad \alpha, a>0, \quad x \in \mathbb{R}$,

with density function

$$
g(x)=\frac{a \alpha \pi}{e^{\alpha}-1} f(x) F(x)^{a-1} \sin \left(\frac{\pi}{2} F(x)^{a}\right)\left(1-\cos \left(\frac{\pi}{2} F(x)^{a}\right)\right) e^{\alpha\left(1-\cos \left(\frac{\pi}{2} F(x)^{a}\right)\right)^{2}}, \quad x \in \mathbb{R} .
$$

\subsection{Another extended Cosine- $X$ family of distributions}

Taking $R(x)=\alpha\left(1-\cos \left(\frac{\pi}{2} F(x)\right)\right)^{2}+\beta\left(1-\cos \left(\frac{\pi}{2} F(x)\right)\right)$ in (14), we define the cdf of the another extended cosine- $X$ (for short 'AEC- $X$ ') family as

$$
G(x)=\frac{e^{\alpha\left(1-\cos \left(\frac{\pi}{2} F(x)\right)\right)^{2}+\beta\left(1-\cos \left(\frac{\pi}{2} F(x)\right)\right)}-1}{e^{\alpha+\beta}-1}, \quad \alpha, \beta>0, \quad x \in \mathbb{R} .
$$

The pdf of the AEC- $X$ family can easily be obtained by simply differentiating (24).

\subsection{Another extended Cosine exponentiated- $X$ family}

Taking $\quad R(x)=\alpha\left(1-\cos \left(\frac{\pi}{2} F(x)^{a}\right)\right)^{2}+\beta\left(1-\cos \left(\frac{\pi}{2} F(x)^{a}\right)\right)$ in (14), we introduce another extended cosine exponentiated- $X$ (for short 'AECE- $X$ ') via the cdf

$G(x)=\frac{e^{\alpha\left(1-\cos \left(\frac{\pi}{2} F(x)^{a}\right)\right)^{2}+\beta\left(1-\cos \left(\frac{\pi}{2} F(x)^{a}\right)\right)}-1}{e^{\alpha+\beta}-1}, \quad \alpha, \beta, a>0, \quad x \in \mathbb{R}$.

By differentiating (25), we get the density function of the AECE- $X$ family.

\subsection{The extended transmuted-G family}

Let $T(x)$ be the cdf of the transmuted distribution family. Then we define the extended transmuted-G family (for short 'ET-G') by taking $R(x)=\alpha T(x)^{2}+\beta T(x)$ in (14), as follows $G(x)=\frac{e^{\alpha T(x)^{2}+\beta T(x)}-1}{e^{\alpha+\beta}-1}, \quad \alpha, \beta, \xi>0, x \in \mathbb{R}$,

\subsection{The extended Kumaraswamy-G family}

Let $K(x)$ be the cdf of the Kumaraswamy distributions. Then, we define the extended Kumaraswamy family (for short ' $\mathrm{EKu}-\mathrm{G}$ ') by taking $R(x)=\alpha K(x)^{2}+\beta K(x)$ in (14), as follows

$$
G(x)=\frac{e^{\alpha K(x)^{2}+\beta K(x)}-1}{e^{\alpha+\beta}-1}, \quad \alpha, \beta, \xi>0, \quad x \in \mathbb{R}
$$

\subsection{The alpha power transformed Cosine- $X$ family}

We define an extended form of the alpha power transformed family by

$G(x)=\frac{\alpha^{R(x)}-1}{\alpha-1}, \quad \alpha>0, \alpha \neq 1, x \in \mathbb{R}$, 
Ahmed, Z., Hamedani, G. G. and Butt, N.S.

where, $R(x)$ may be any function of cdf satisfying the conditions stated in section 2. Here, we define a new family, called the alpha power transformed cosine- $X$ (for short 'APTC$\left.X^{\prime}\right)$ family by taking $R(x)=1-\cos \left(\frac{\pi}{2} F(x)\right)$ in (28).

$G(x)=\frac{\alpha^{1-\cos \left(\frac{\pi}{2} F(x)\right)}-1}{\alpha-1}, \quad \alpha>0, \alpha \neq 1, x \in \mathbb{R}$.

The pdf of the APTC- $X$ can easily be obtained by simply differentiating (29).

\subsection{The alpha power transformed Cosine exponentiated- $X$ family}

A random variable $X$ is said to have the alpha power transformed cosine exponentiated- $X$ (for short 'APTCE- $X$ ') family, if its cdf is given by

$G(x)=\frac{\alpha^{1-\cos \left(\frac{\pi}{2} F(x)^{a}\right)}-1}{\alpha-1}, \quad \alpha, a>0, \alpha \neq 1, x \in \mathbb{R}$,

with density function

$$
g(x)=\frac{a \pi}{2(\alpha-1)} f(x) F(x)^{a-1} \sin \left(\frac{\pi}{2} F(x)^{a}\right) \alpha^{1-\cos \left(\frac{\pi}{2} F(x)^{a}\right)}, \quad x \in \mathbb{R} .
$$

\subsection{The extended alpha power transformed- $X$ family}

Taking $R(x)=\alpha_{1} F(x)^{2}+\beta F(x)$ in (28), we introduce the extended alpha power transformed$X$ (for short 'EAPT- $X$ ') via the cdf

$G(x)=\frac{\alpha^{\alpha_{1} F(x)^{2}+\beta F(x)}-1}{\alpha-1}, \quad \alpha, \alpha_{1}, \beta>0, \alpha \neq 1, x \in \mathbb{R}$.

By differentiating (33), we get the density function of the EAPT- $X$ family.

\section{Characterization Results}

In designing a stochastic model for a particular modeling problem, an investigator will be vitally interested to know if their model fits the requirements of a specific underlying probability distribution. To this end, the investigator will rely on the characterizations of the selected distribution. Thus, the problem of characterizing a distribution is an important problem in various fields and has recently attracted the attention of many researchers. Consequently, various characterization results have been reported in the literature. These characterizations have been established in different directions. This section deals with various characterizations of 12 proposed distributions listed in Section 3. These characterizations are based on a simple relationship between two truncated moments. It should be mentioned that one important advantage of our characterization is that the cdf need not have a closed, and moreover, it depends on the solution of a first order differential equation, which provides a bridge between probability and differential equation. In the subsection 4.1 we provide the characterizations of the Extended Zubair-G (EZ-G) family of distributions. Similar characterizations can be stated for the other 11 distributions.

\subsection{Characterizations based on two truncated moments}


This subsection deals with the characterizations of the EZ-G distribution based on the ratio of two truncated moments. Our first characterization employs a theorem of Glänzel (1987); see Theorem 1 of Appendix A.

Proposition 4.1. Let $X: \Omega \rightarrow \mathbb{R}$ be a continuous random variable and let $q_{1}(x) \equiv 1$ and $q_{2}(x)=e^{\alpha F(x)^{2}+\beta F(x)}$ for $x \in \mathbb{R}$. Then, the random variable $X$ has pdf (12) if and only if the function $\eta$ defined in Theorem 1 is of the form

$$
\eta(x)=\frac{1}{2}\left(e^{\alpha+\beta}+e^{\alpha F(x)^{2}+\beta F(x)}\right), \quad x \in \mathbb{R} .
$$

Proof. Suppose the random variable $X$ has pdf (12), then

$$
(1-F(x)) E\left(q_{1}(X) \mid X \geq x\right)=\frac{1}{e^{\alpha+\beta}-1}\left(e^{\alpha+\beta}-e^{\alpha F(x)^{2}+\beta F(x)}\right), \quad x \in \mathbb{R},
$$

and

$$
(1-F(x)) E\left(q_{2}(X) \mid X \geq x\right)=\frac{1}{2\left(e^{\alpha+\beta}-1\right)}\left(e^{2(\alpha+\beta)}-e^{2\left(\alpha F(x)^{2}+\beta F(x)\right)}\right), \quad x \in \mathbb{R} .
$$

Further,

$$
\eta(x) q_{1}(x)-q_{2}(x)=\frac{1}{2}\left(e^{\alpha+\beta}-e^{\alpha F(x)^{2}+\beta F(x)}\right)>0, \quad \text { for } x \in \mathbb{R} .
$$

Conversely, if $\eta$ is of the above form, then

$$
s^{\prime}(x)=\frac{\eta^{\prime}(x) q_{1}(x)}{\eta(x) q_{1}(x)-q_{2}(x)}=\frac{f(x)(2 \alpha F(x)+\beta) e^{\alpha F(x)^{2}+\beta F(x)}}{e^{\alpha+\beta}-e^{\alpha F(x)^{2}+\beta F(x)}}, \quad x \in \mathbb{R},
$$

and hence

$$
s(x)=-\log \left(e^{\alpha+\beta}-e^{\alpha F(x)^{2}+\beta F(x)}\right), \quad x \in \mathbb{R} .
$$

Now, in view of Theorem $1, X$ has density (12).

Corollary 4.1. Let $X: \Omega \rightarrow \mathbb{R}$ be a continuous random variable and let $q_{1}(x)$ be as in Proposition 4.1. The random variable $X$ has pdf (12) if and only if there exist functions $q_{2}$ and $\eta$ defined in Theorem 1 satisfying the following differential equation

$$
\frac{\eta^{\prime}(x) q_{1}(x)}{\eta(x) q_{1}(x)-q_{2}(x)}=\frac{f(x)(2 \alpha F(x)+\beta) e^{\alpha F(x)^{2}+\beta F(x)}}{e^{\alpha+\beta}-e^{\alpha F(x)^{2}+\beta F(x)}}, \quad x \in \mathbb{R} .
$$

Corollary 4.2. The general solution of the differential equation in Corollary 4.1 is

$$
\eta(x)=\left(e^{\alpha+\beta}-e^{\alpha F(x)^{2}+\beta F(x)}\right)\left[-\int \frac{f(x)(2 \alpha F(x)+\beta) e^{\alpha F(x)^{2}+\beta F(x)}}{(1-F(x ; \xi))^{\alpha+1}}\left(q_{1}(x)\right)^{-1} q_{2}(x) d x+D\right],
$$

where $D$ is a constant. Note that a set of functions satisfying the above differential equation is given in Proposition 4.1 with $D=1 / 2$. However, it should also be noted that there are other triplets $\left(q_{1}(x), q_{2}(x), \eta(x)\right)$ satisfying the conditions of Theorem 1 .

\section{Concluding Remarks}

The need of compounding and generalizing distributions were first felt in the financial and actuarial science and later in many other fields which researchers adopted this approach 
for lifetime and reliability modeling. In this way, the possible available compound and generalized G-classes are surveyed and using these basic principles nearly 12 new classes are proposed. The goal of providing a variety of new class classes is to test the flexibility of the proposed models to cope with the data available in complex situations. The parameters inducted in this way might be helpful in describing the phenomenon generated from real-lifetime data sets. We expect that these distributions will be an addition to the art of constructing useful probability models. One can imagine its motivation and usefulness in the fields which are not touched so far. Lastly, we offer more choices to the learners and practitioners of modeling to compare different models and to illustrate usefulness of old and new classes of distributions.

\section{References}

1. Abdel Rahman, N. S. and El-Bassiouny, A.H. (2017). On the alpha-power exponential Weibull distribution, The 2nd National of Mathematics and Its Applications (NCMA17), 1-18.

2. Abdul-Moniem, I. B. (2012). Recurrence relations for moments of lower generalized order statistics from exponentiated Lomax distribution and its characterization. Journal of Mathematical and Computational Science, 2(4), 999-1011.

3. Afify, A. Z., Cordeiro, G. M., Yousof, H. M., Alzaatreh, A., \& Nofal, Z. M. (2016). The Kumaraswamy transmuted-G family of distributions: properties and applications. Journal of Data Science, 14(2), 245-270.

4. Afify, A. Z., Cordeiro, G. M., Yousof, H. M., Saboor, A., \& Ortega, E. M. (2016). The Marshall-Olkin additive Weibull distribution with variable shapes for the hazard rate. Hacettepe Journal of Mathematics and Statistics, Forthcoming.

5. Afify, A. Z., Yousof, H. M., Hamedani, G. G., \& Aryal, G. (2016). The exponentiated Weibull-Pareto distribution with application. J. Stat. Theory Appl, 15, 328-346.

6. Ahmad, Z. (2018). The Zubair-G Family of Distributions: Properties and Applications. Annals of Data Science, 1-14.

7. Ahmad, Z., Elgarhy, M., \& Hamedani, G. G. (2018). A new Weibull-X family of distributions: properties, characterizations and applications. Journal of Statistical Distributions and Applications, 5(1), 5.

8. Akinsete, A., Famoye, F., \& Lee, C. (2014). The Kumaraswamy-geometric distribution. Journal of statistical distributions and applications, 1(1), 17.

9. Al-Babtain, A., Fattah, A. A., Ahmed, A. H. N., \& Merovci, F. (2017). The Kumaraswamy-transmuted exponentiated modified Weibull distribution. Communications in Statistics-Simulation and Computation, 46(5), 3812-3832. 
10. Alexander, C., Cordeiro, G. M., Ortega, E. M., \& Sarabia, J. M. (2012). Generalized beta-generated distributions. Computational Statistics \& Data Analysis, 56(6), 1880-1897.

11. AL-Fattah, A. M., EL-Helbawy, A. A., \& AL-Dayian, G. R. (2017). INVERTED KUMARASWAMY DISTRIBUTION: PROPERTIES AND ESTIMATION. Pakistan Journal of Statistics, 33(1).

12. Alice, T., \& Jose, K. K. (2003). Marshall-Olkin Pareto processes. Far East Journal of Theoretical Statistics, 9(2), 117-132.

13. Alice, T., \& Jose, K. K. (2005). Marshall-Olkin logistic processes. STARS Int J, 6, 1-11.

14. Alizadeh, M., Emadi, M., Doostparast, M., Cordeiro, G. M., Ortega, E. M., \& Pescim, R. R. (2015). A new family of distributions: the Kumaraswamy odd log-logistic, properties and applications. Hacettepa Journal of Mathematics and Statistics, forthcomig.

15. Alizadeh, M., Tahir, M. H., Cordeiro, G. M., Mansoor, M., Zubair, M., \& Hamedani, G. G. (2015). The Kumaraswamy Marshal-Olkin family of distributions. Journal of the Egyptian Mathematical Society, 23(3), 546-557.

16. Al-Saiari, A. Y., Baharith, L. A., \& Mousa, S. A. (2014). Marshall-Olkin extended Burr type XII distribution. International Journal of Statistics and probability, 3(1), 78.

17. Alzaatreh, A., Lee, C., \& Famoye, F. (2013). A new method for generating families of continuous distributions. Metron, 71(1), 63-79.

18. Alzaghal, A., Famoye, F., \& Lee, C. (2013). Exponentiated T-X family of distributions with some applications. International Journal of Statistics and Probability, 2(3), 31.

19. Andrade, T., Rodrigues, H., Bourguignon, M., \& Cordeiro, G. (2015). The exponentiated generalized Gumbel distribution. Revista Colombiana de Estadística, 38(1), 123-143.

20. Aryal, G., \& Elbatal, I. (2015). Kumaraswamy modified inverse Weibull distribution: Theory and application. Applied Mathematics \& Information Sciences, 9(2), 651.

21. Aryal, G., \& Elbatal, I. (2015). On the exponentiated generalized modified Weibull distribution. Communications for Statistical Applications and Methods, 22(4), 333-348.

22. Ashour, S. K., \& Eltehiwy, M. A. (2015). Exponentiated power Lindley distribution. Journal of advanced research, 6(6), 895-905.

23. Ashour, S. K., \& Eltehiwy, M. A. (2015). Exponentiated power Lindley distribution. Journal of advanced research, 6(6), 895-905.

24. Aslam, M., Hussain, Z., \& Asghar, Z. (2018). Cubic transmuted-g family of distributions and its properties. Stochastics and Quality Control, 33(2), 103112. 
25. Batal, I. E. L., \& Kareem, A. (2014). Statistical properties of Kumaraswamy exponentiated Lomax distribution. Journal of modern Mathematics and statistics, 8(1), 1-7.

26. Behairy, S. M., AL-Dayian, G. R., \& EL-Helbawy, A. A. (2016). The Kumaraswamy-Burr type III distribution: properties and estimation. British Journal of Mathematics, 14, 1-21.

27. Benkhelifa, L. (2016). The Marshall-Olkin extended generalized Gompertz distribution. arXiv preprint arXiv:1603.08242.

28. Cakmakyapan, S., Ozel, G., El Gebaly, Y. M. H., \& Hamedani, G. G. (2018). The Kumaraswamy Marshall-Olkin Log-Logistic Distribution with Application. Journal of Statistical Theory and Applications, 17(1), 59-76.

29. Chhetri, S. B., Akinsete, A. A., Aryal, G., \& Long, H. (2017). The Kumaraswamy transmuted Pareto distribution. Journal of Statistical Distributions and Applications, 4(1), 11.

30. Chukwu, A. U., \& Ogunde, A. A. (2016). On Kumaraswamy Gompertz Makeham distribution. American Journal of Mathematics and Statistics, 6(3), 122-127.

31. Cordeiro, G. M., \& Lemonte, A. J. (2013). On the Marshall-Olkin extended Weibull distribution. Statistical papers, 54(2), 333-353.

32. Cordeiro, G. M., Alizadeh, M., \& Ortega, E. M. (2014). The exponentiated halflogistic family of distributions: Properties and applications. Journal of Probability and Statistics, 2014.

33. Cordeiro, G. M., Alizadeh, M., Nascimento, A. D., \& Rasekhi, M. (2016). The Exponentiated Gompertz Generated Family of Distributions: Properties and Applications. Chilean Journal of Statistics (ChJS), 7(2).

34. Cordeiro, G. M., Nadarajah, S., \& Ortega, E. M. (2012). The Kumaraswamy Gumbel distribution. Statistical Methods \& Applications, 21(2), 139-168.

35. Cordeiro, G. M., Ortega, E. M., \& da Cunha, D. C. (2013). The exponentiated generalized class of distributions. Journal of Data Science, 11(1), 1-27.

36. Cordeiro, G. M., Ortega, E. M., \& Nadarajah, S. (2010). The Kumaraswamy Weibull distribution with application to failure data. Journal of the Franklin Institute, 347(8), 1399-1429.

37. Cordeiro, G. M., Ortega, E. M., \& Silva, G. O. (2011). The exponentiated generalized gamma distribution with application to lifetime data. Journal of statistical computation and simulation, 81(7), 827-842.

38. Cordeiro, G. M., Ortega, E. M., \& Silva, G. O. (2014). The Kumaraswamy modified Weibull distribution: theory and applications. Journal of Statistical Computation and Simulation, 84(7), 1387-1411.

39. Cordeiro, G. M., Saboor, A., Khan, M. N., Ozel, G., \& Pascoa, M. A. (2016). The Kumaraswamy Exponential-Weibull Distribution: Theory and 
Applications. Hacettepe journal of mathematics and statistics, 45(4), 12031229.

40. da Silva, R. V., Gomes-Silva, F., Ramos, M. W. A., \& Cordeiro, G. M. (2015). The exponentiated Burr XII Poisson distribution with application to lifetime data. International Journal of Statistics and Probability, 4(4), 112.

41. De Pascoa, M. A., Ortega, E. M., \& Cordeiro, G. M. (2011). The Kumaraswamy generalized gamma distribution with application in survival analysis. Statistical methodology, 8(5), 411-433.

42. De Santana, T. V. F., Ortega, E. M., Cordeiro, G. M., \& Silva, G. O. (2012). The Kumaraswamy-log-logistic distribution. Journal of Statistical Theory and Applications, 11(3), 265-291.

43. Dey, S., Ghosh, I., \& Kumar, D. (2018). Alpha-Power Transformed Lindley Distribution: Properties and Associated Inference with Application to Earthquake Data. Annals of Data Science, 1-28.

44. Dey, S., Nassar, M., \& Kumar, D. (2019). Alpha power transformed inverse Lindley distribution: A distribution with an upside-down bathtub-shaped hazard function. Journal of Computational and Applied Mathematics, 348, 130-145.

45. El-Bassiouny, A. H., El-Damcese, M., Mustafa, A., \& Eliwa, M. S. (2017). Exponentiated generalized Weibull-Gompertz distribution with application in survival analysis. J. Stat. Appl. Probab, 6, 7-16.

46. Elbatal, I. (2013). Kumaraswamy generalized linear failure rate distribution. Indian J Comput Appl Math, 1(1), 61-78.

47. Elbatal, I. (2013). The Kumaraswamy exponentiated Pareto distribution. Economic Quality Control, 28(1), 1-8.

48. El-Damcese, M. A., Mustafa, A., El-Desouky, B. S., \& Mustafa, M. E. (2016). The Kumaraswamy flexible Weibull extension. rn, 55, 7.

49. El-Gohary, A., El-Bassiouny, A. H., \& El-Morshedy, M. (2015). Exponentiated flexible Weibull extension distribution. rn, 55, 7.

50. Eugene, N., Lee, C., \& Famoye, F. (2002). Beta-normal distribution and its applications. Communications in Statistics-Theory and methods, 31(4), 497512.

51. Ghitany, M. E. (2005). Marshall-Olkin extended Pareto distribution and its application. International Journal of Applied Mathematics, 18(1), 17.

52. Ghitany, M. E., Al-Awadhi, F. A., \& Alkhalfan, L. A. (2007). Marshall-Olkin extended Lomax distribution and its application to censored data. Communications in Statistics-Theory and Methods, 36(10), 1855-1866.

53. Ghitany, M. E., Al-Hussaini, E. K., \& Al-Jarallah, R. A. (2005). MarshallOlkin extended Weibull distribution and its application to censored data. Journal of Applied Statistics, 32(10), 1025-1034. 
54. Glänzel, W. (1987). A characterization theorem based on truncated moments and its application to some distribution families. In Mathematical statistics and probability theory (pp. 75-84). Springer, Dordrecht.

55. Glänzel, W., \& Hamedani, G. G. (2001). Characterizations of univariate continuous distributions. Studia Scientiarum Mathematicarum Hungarica, 37(1-2), 83-118.

56. Gomes, A. E., da-Silva, C. Q., Cordeiro, G. M., \& Ortega, E. M. (2014). A new lifetime model: the Kumaraswamy generalized Rayleigh distribution. Journal of statistical computation and simulation, 84(2), 290-309.

57. Granzotto, D. C. T., Louzada, F., \& Balakrishnan, N. (2017). Cubic rank transmuted distributions: inferential issues and applications. Journal of Statistical Computation and Simulation, 87(14), 2760-2778.

58. Gui, W. (2013). A Marshall-Olkin power Log-Normal distribution and its applications to survival data. International Journal of Statistics and Probability, 2(1), 63.

59. Gui, W. (2013). Marshall-Olkin extended log-logistic distribution and its application in minification processes. Appl Math Sci, 7(80), 3947-3961.

60. Gupta, R. D., \& Kundu, D. (2001). Exponentiated exponential family: an alternative to gamma and Weibull distributions. Biometrical Journal: Journal of Mathematical Methods in Biosciences, 43(1), 117-130.

61. Haq, M. A. (2016). Kumaraswamy Exponentiated Inverse Rayleigh Distribution. Math. Theo. Model, 6(3), 93-104.

62. Hassan, A. S., \& Abd-Allah, M. (2018). Exponentiated Weibull-Lomax Distribution: Properties and Estimation. Journal of Data Science, 277, 298.

63. Hassan, A. S., \& Abdelghafar, M. A. (2017). Exponentiated Lomax geometric distribution: Properties and applications. Pakistan Journal of Statistics and Operation Research, 13(3), 545-566.

64. Hassan, A. S., \& Elgarhy, M. (2016). Kumaraswamy Weibull-generated family of distributions with applications. Advances and Applications in Statistics, 48(3), 205.

65. Hassan, A. S., Elgarhy, M., Mohamd, R. E., \& Alrajhi, S. (2019). On the Alpha Power Transformed Power Lindley Distribution. Journal of Probability and Statistics, 2019.

66. Hibatullah, R., Widyaningsih, Y., \& Abdullah, S. (2018, October). MarshallOlkin extended inverse power Lindley distribution with applications. In AIP Conference Proceedings (Vol. 2021, No. 1, p. 060025). AIP Publishing.

67. Huang, S., \& Oluyede, B. O. (2014). Exponentiated Kumaraswamy-Dagum distribution with applications to income and lifetime data. Journal of Statistical Distributions and Applications, 1(1), 8. 
68. Jamal, F., Tahir, M. H., Alizadeh, M., \& Nasir, M. A. (2017). On MarshallOlkin Burr X family of distribution. Tbilisi Mathematical Journal, 10(4), 175199.

69. Jan, R., Jan, T. R., \& Ahmad, P. B. (2018). Exponentiated Inverse Power Lindley Distribution and its Applications. arXiv preprint arXiv:1808.07410.

70. Jose, K. K., \& Krishna, E. (2011, October). Marshall-Olkin extended uniform distribution. In ProbStat Forum (Vol. 4, No. October, pp. 78-88).

71. Jose, K. K., Joseph, A., \& Ristić, M. M. (2009). A Marshall-Olkin beta distribution and its applications. Journal of Probability and Statistical Science, 7(2), 173-186.

72. Jose, K. K., Naik, S. R., \& Ristić, M. M. (2010). Marshall-Olkin q-Weibull distribution and max-min processes. Statistical papers, 51(4), 837-851.

73. Khan, M. S., King, R., \& Hudson, I. L. (2018). Kumaraswamy exponentiated Chen distribution for modelling lifetime data. Appl. Math, 12(3), 617-623.

74. Kumar, D. (2016). Ratio and inverse moments of Marshall-Olkin extended Burr type XII distribution based on lower generalized order statistics. Journal of Data Science, 14(1), 53-66.

75. Kumar, D., Kumar, M., Saran, J., \& Jain, N. (2017). The Kumaraswamy-Burr III distribution based on upper record values. American Journal of Mathematical and Management Sciences, 36(3), 205-228.

76. Kumaraswamy, P. (1980). A generalized probability density function for double-bounded random processes. Journal of Hydrology, 46(1-2), 79-88.

77. Kyurkchiev, N., Iliev, A., \& Rahnev, A. (2019). Comments on a Zubair-G family of cumulative lifetime distributions. Some extensions. Communications in applied analysis, 23(1), 1-20.

78. Lemonte, A. J., Barreto-Souza, W., \& Cordeiro, G. M. (2013). The exponentiated Kumaraswamy distribution and its log-transform. Brazilian Journal of Probability and Statistics, 27(1), 31-53.

79. Lepetu, L., Oluyede, B. O., Makubate, B., Foya, S., \& Mdlongwa, P. (2017). Marshall-Olkin Log-Logistic Extended Weibull Distribution: Theory, Properties and Applications. Journal of Data Science, 15(4).

80. Mahdavi, A., \& Kundu, D. (2017). A new method for generating distributions with an application to exponential distribution. Communications in StatisticsTheory and Methods, 46(13), 6543-6557.

81. Marshall, A. W., \& Olkin, I. (1997). A new method for adding a parameter to a family of distributions with application to the exponential and Weibull families. Biometrika, 84(3), 641-652.

82. Merovci, F. A. T. O. N., \& Sharma, V. K. (2014). The Kumaraswamy-Lindley Distribution: Properties and Applications. 
83. Mudholkar, G. S., \& Srivastava, D. K. (1993). Exponentiated Weibull family for analyzing bathtub failure-rate data. IEEE transactions on reliability, 42(2), 299-302.

84. Muhammad, M., Muhammad, I., \& Yaya, A. M. (2018). The Kumaraswamy Exponentiated U-Quadratic Distribution: Properties and Application. Asian Journal of Probability and Statistics, 1(3), 1-17.

85. Mustafa, A., El-Desouky, B. S., \& AL-Garash, S. (2016). The Marshall-Olkin Flexible Weibull Extension Distribution. arXiv preprint arXiv:1609.08997.

86. Nadarajah, S. (2005). Exponentiated beta distributions. Computers \& Mathematics with Applications, 49(7-8), 1029-1035.

87. Nadarajah, S. (2005). Exponentiated Pareto distributions. Statistics, 39(3), 255260.

88. Nadarajah, S. (2006). The exponentiated Gumbel distribution with climate application. Environmetrics: The official journal of the International Environmetrics Society, 17(1), 13-23.

89. Nadarajah, S., \& Eljabri, S. (2013). The Kumaraswamy GP distribution. Journal of Data Science, 11(4), 739-766.

90. Nadarajah, S., \& Gupta, A. K. (2007). The exponentiated gamma distribution with application to drought data. Calcutta Statistical Association Bulletin, 59(12), 29-54.

91. Nadarajah, S., \& Kotz, S. (2006). The exponentiated type distributions. Acta Applicandae Mathematica, 92(2), 97-111.

92. Nasir, A., Bakouch, H. S., \& Jamal, F. (2018). Kumaraswamy odd Burr G family of distributions with applications to reliability data. Studia Scientiarum Mathematicarum Hungarica, 55(1), 94-114.

93. Nasiru, S., Mwita, P. N. and Ngesa, O. (2019). Alpha Power Transformed Frechet Distribution. Applied Mathematics \& Information Sciences, 13(1):129141.

94. Nassar, M. M. (2016). The Kumaraswamy Laplace Distribution. Pakistan Journal of Statistics and Operation Research, 12(4), 609-624.

95. Nofal, Z. M., \& Abd El Hadi, N. E. (2015). Exponentiated Transmuted Generalized Raleigh Distribution: A New Four Parameter Rayleigh Distribution. Pakistan journal of statistics and operation research, 11(1), 115134.

96. Oguntunde, P. E., Adejumo, A., \& Owoloko, E. A. (2017). Application of Kumaraswamy Inverse Exponential Distribution to Real Lifetime Data. International Journal of Applied Mathematics and Statistics.

97. Okorie, I. E., Akpanta, A. C., \& Ohakwe, J. (2016). The exponentiated Gumbel type-2 distribution: properties and application. International Journal of Mathematics and Mathematical Sciences, 2016. 
98. Okorie, I. E., Akpanta, A. C., \& Ohakwe, J. (2017). Marshall-Olkin generalized Erlang-truncated exponential distribution: Properties and applications. Cogent Mathematics, 4(1), 1285093.

99. Pakungwati, R. M., Widyaningsih, Y., \& Lestari, D. (2018, November). Marshall-Olkin Extended Inverse Weibull Distribution and Its Application. In Journal of Physics: Conference Series (Vol. 1108, No. 1, p. 012114). IOP Publishing.

100. Paranaíba, P. F., Ortega, E. M., Cordeiro, G. M., \& Pascoa, M. A. D. (2013). The Kumaraswamy Burr XII distribution: theory and practice. Journal of Statistical Computation and Simulation, 83(11), 2117-2143.

101. Pararai, M., Oluyedede, B. O., \& Warahena-Liyanage, G. (2015). Kumaraswamy Lindley-Poisson distribution: theory and applications. Asian Journal of Mathematics and Applications, 2015.

102. Pavlov, N., Kyurkchiev, N., Iliev, A., Rahnev, A. and Rahven, A. (2019). A Note on the Zubair-G Family with baseline Lomax Cumulative Distribution Function. Some Applications. communications in applied analysis, 120(3):471486.

103. Pereira, M. B., Silva, R. B., Zea, L. M., \& Cordeiro, G. M. (2012). The Kumaraswamy Pareto distribution. arXiv preprint arXiv:1204.1389.

104. Pérez-Casany, M., \& Casellas, A. (2013). Marshall-Olkin Extended Zipf Distribution. arXiv preprint arXiv:1304.4540.

105. Pogány, T. K., Saboor, A., \& Provost, S. (2015). The Marshall-Olkin exponential Weibull distribution. Hacettepe Journal of Mathematics and Statistics, 44(6), 1579.

106. Rahman, M. M., Al-Zahrani, B., \& Shahbaz, M. Q. (2018). A General Transmuted Family of Distributions. Pakistan Journal of Statistics and Operation Research, 14(2).

107. Rahneva, O., Terzieva, T. and Golev, A. (2019). Investigations on the Zubairfamily with baseline Ghosh-Bourguignon's extended Burr XII cumulative sigmoid. some applications. Neural, Parallel, and Scientific Computations, 27(1), 11-22.

108. Ramadan and Walaa (2018). On the Alpha-Power Inverse Weibull Distribution. International Journal of Computer Applications, 181(11),

109. Ramos, M. W. A., Marinho, P. R. D., da Silva, R. V., \& Cordeiro, G. M. (2013). The exponentiated Lomax Poisson distribution with an application to lifetime data. Advances and Applications in Statistics, 34(2), 107-135.

110. Rao, G. S., Ghitany, M. E., \& Kantam, R. R. L. (2011). An economic reliability test plan for Marshall-Olkin extended exponential distribution. Applied Mathematical Sciences, 5(3), 103-112. 
111. Ristić, M. M., \& Balakrishnan, N. (2012). The gamma-exponentiated exponential distribution. Journal of Statistical Computation and Simulation, 82(8), 1191-1206.

112. Ristić, M. M., \& Kundu, D. (2015). Marshall-Olkin generalized exponential distribution. Metron, 73(3), 317-333.

113. Ristic, M. M., Jose, K. K., \& Ancy, J. (2007). A Marshall-Olkin gamma distribution and minification process. Stress Anxiety Res Soc, 11, 107-117.

114. Roges, D. L., de Gusmao, F., \& Diniz, C. (2014). The Kumaraswamy inverse Rayleigh distribution. Unpublished manuscript.

115. Saboor, A., \& Pogány, T. K. (2016). Marshall-Olkin gamma-Weibull distribution with applications. Communications in Statistics-Theory and Methods, 45(5), 1550-1563.

116. Sandhya, E., \& Prasanth, C. B. (2014). Marshall-Olkin discrete uniform distribution. Journal of probability, 2014.

117. Santos-Neto, M., Bourguignon, M., Zea, L. M., Nascimento, A. D., \& Cordeiro, G. M. (2014). The Marshall-Olkin extended Weibull family of distributions. Journal of Statistical Distributions and Applications, 1(1), 9.

118. Sarhan, A. M., \& Apaloo, J. (2013). Exponentiated modified Weibull extension distribution. Reliability Engineering \& System Safety, 112, 137-144.

119. Saulo, H., Leão, J., \& Bourguignon, M. (2012). The Kumaraswamy BirnbaumSaunders Distribution. Journal of Statistical Theory and Practice, 6(4), 745759.

120. Selim, M. A., \& Badr, A. M. (2016). The Kumaraswamy generalized power Weibull distribution. Math. Theo. Model, 6, 110-124.

121. Shahbaz, M. Q., Shahbaz, S., \& Butt, N. S. (2012). The Kumaraswamy-inverse Weibull distribution. Pakistan journal of statistics and operation research, 8(3), 479-489.

122. Shams, T. M. (2013). The Kumaraswamy-generalized exponentiated Pareto distribution. European Journal of Applied Sciences, 5(3), 92-99.

123. Shams, T. M. (2013). The Kumaraswamy-generalized Lomax distribution. Middle-East Journal of Scientific Research, 17(5), 641-646.

124. Shaw, W. T., \& Buckley, I. R. (2009). The alchemy of probability distributions: beyond Gram-Charlier expansions, and a skew-kurtotic-normal distribution from a rank transmutation map. arXiv preprint arXiv:0901.0434.

125. Shirke, D. T., \& Kakade, C. S. (2006). On exponentiated lognormal distribution. International Journal of Agricultural and Statistical Sciences, 2(2), 319-326.

126. Tahir, M. H., \& Cordeiro, G. M. (2016). Compounding of distributions: a survey and new generalized classes. Journal of Statistical Distributions and Applications, 3(1), 13. 
127. Tahir, M. H., Cordeiro, G. M., Alzaatreh, A., Mansoor, M., \& Zubair, M. (2016). The Logistic-X family of distributions and its applications. Communications in Statistics-Theory and Methods, 45(24), 73267349.

128. Thomas, A., \& Jose, K. K. (2005). Marshall-Olkin semi-Weibull minification processes. Recent Adv Stat Theory Appl, 1, 6-17.

129. Torabi, H., \& Hedesh, N. M. (2012). The gamma-uniform distribution and its applications. Kybernetika, 48(1), 16-30.

130. Torabi, H., \& Montazeri, N. H. (2014). The logistic-uniform distribution and its applications. Communications in Statistics-Simulation and Computation, 43(10), 2551-2569.

131. Unal, C., Cakmakyapan, S., \& Ozel, G. (2018). Alpha Power Inverted Exponential Distribution: Properties and Application. Gazi University Journal of Science, 31(3).

132. Usman, R. M., Haq, M. A., \& Talib, J. (2017). Kumaraswamy half-logistic distribution: properties and applications. J Stat Appl Probab, 6, 597-609.

133. Yeğen, D., \& Özel, G. (2018). Marshall-Olkin Half Logistic Distribution with Theory and Applications. Alphanumeric Journal, 6(2), 407-416.

134. Yousof, H. M., Afify, A. Z., Nadarajah, S., Hamedani, G., \& Aryal, G. R. (2018). The Marshall-Olkin generalized-G family of distributions with Applications. Statistica, 78(3), 273-295.

135. Zografos, K., \& Balakrishnan, N. (2009). On families of beta-and generalized gamma-generated distributions and associated inference. Statistical Methodology, 6(4), 344-362.

\section{Appendix A.}

Theorem 1. Let $(\Omega, F, P)$ be a given probability space and let $H=[d ; e]$ be an interval for some $d<e(d=-\infty ; e=\infty$ might as well be allowed). Let $X: \Omega \rightarrow H$ be a continuous random variable with the distribution function $F$ and let $q_{1}(x)$ and $q_{2}(x)$ be two real functions defined on $H$ such that

$$
E\left(q_{2}(X) \mid X \geq x\right)=E\left(q_{1}(X) \mid X \geq x\right) \eta(x), \quad x \in H,
$$

is defined with some real function $\eta$. Assume that $q_{1}, q_{2} \in C^{1}(H), \eta \in C^{2}(H)$ and $F$ is twice continuously differentiable and strictly monotone function on the set $H$. Finally, assume that the equation $\xi q_{1}=q_{2}$ has no real solution in the interior of $H$. Then $F$ is uniquely determined by the functions $q_{1}, q_{2}$ and $\eta$ particularly

$$
F(x)=\int_{a}^{x} C\left|\frac{\eta^{\prime}(u)}{\eta(u) q_{1}(u)-q_{2}(u)}\right| \exp (-s(u)) d u,
$$


where the function $s(u)$ is a solution of the differential equation $s^{\prime}=\frac{\eta^{\prime} q_{1}}{\eta q_{1}-q_{2}}$ and $C$ is the normalization constant, such that $\int_{H} d F=1$.

Note that the result, however, holds also when the interval $H$ is not closed, since the condition is on the interior of $H$.

We like to mention that this kind of characterization based on the ratio of truncated moments is stable in the sense of weak convergence (see, Glänzel (1990)), in particular, let us assume that there is a sequence $\left\{X_{n}\right\}$ of random variables with distribution functions $\left\{F_{n}\right\}$ such that the functions $q_{1 n}, q_{2 n}$ and $\eta_{n}(n \in \mathbb{N})$ satisfy the conditions of Theorem 1 and let $q_{1 n} \rightarrow q_{1}, q_{2 n} \rightarrow q_{2}$ for some continuously differentiable real functions $q_{1}$ and $q_{2}$. Let, finally, $X$ be a random variable with distribution $F(x)$. Under the condition that $q_{1 n}$ and $q_{2 n}$ are uniformly integrable and the family $\left\{F_{n}\right\}$ is relatively compact, the sequence $X_{n}$ converges to $X$ in distribution if and only if $\eta_{n}$ converges to $\eta$, where

$$
\eta(x)=\frac{E\left(q_{2}(X) \mid X \geq x\right)}{E\left(q_{1}(X) \mid X \geq x\right)} .
$$

This stability theorem makes sure that the convergence of distribution functions is reflected by corresponding convergence of the functions $q_{1}, q_{2}$ and $\eta$ respectively. It guarantees, for instance, the 'convergence' of characterization of the Wald distribution to that of the LevySmirnov distribution if $\alpha \rightarrow \infty$ as was pointed out in Glänzel and Hamedani (2001).

A further consequence of the stability property of Theorem 1 is the application of this theorem to special tasks in statistical practice such as the estimation of the parameters of discrete distributions. For such purpose, the functions $q_{1}, q_{2}$ and, specially, $\eta$ should be as simple as possible. Since the function triplet is not uniquely determined it is often possible to choose $\eta$ as a linear function. Therefore, it is worth analyzing some special cases which helps to find new characterizations reflecting the relationship between individual continuous univariate distributions and appropriate in other areas of statistics. 